\title{
Elektrokleingeräte - Von der Sammlung zur Sekundärressource
}

\author{
Nicole Unger · Peter Beigl · Stefan Salhofer
}

Online publiziert: 11. September 2017

(c) Der/die Autor(en) 2017. Dieser Artikel ist eine Open-Access-Publikation.

\begin{abstract}
Zusammenfassung Elektroaltgeräte stellen mit einem jährlichen Zuwachs von 3 bis $5 \%$ einen der am schnellsten wachsenden Abfallströme in Europa dar. Vorgaben und Ambitionen der Europäischen Union zielen auf die Vermeidung des Anfalls von Elektroaltgeräten, eine nachhaltige Ressourcennutzung sowie eine Beschränkung von gefährlichen Stoffen ab. Im Rahmen eines FFG-Forschungsprojekts wurden die Materialströme von Elektrokleingeräten entlang der gesamten Verwer-

hend die größten Verbesserungspotenziale) treten in der Sammlung $(41 \%$ Verluste) und in der stofflichen Verwertung (30 bis $36 \%$ Verluste) auf. Die Recherche von Sammelsystemen zeigt, dass die Rücknahme durch den Handel sowie die Sammlung im öffentlichen Raum vielversprechende Beiträge zur Erhöhung der Sammelmengen liefern können. Die modellbasierte Outputschätzung zeigte für Leiterplatten, Eisen und Kunststoffe eine zufriedenstellende Genauigkeit.
\end{abstract} tungskette und die dabei auftretenden Materialverluste von der Sammlung bis zur Sekundärressource untersucht. Dabei wurden neue Ansätze zur Sammlung von Elektrokleingeräten aus Haushalten recherchiert sowie ein stochastisches Modell zur Abschätzung der Materialzusammensetzung des Outputs einer mechanischen Behandlungsanlage basierend auf dem Inputmaterial (Sammelware) entwickelt. Primärdaten wurden in Kooperation mit dem Betreiber einer österreichischen mechanischen Aufbereitungsanlage im Rahmen von Batchversuchen sowohl für das Inputmaterial in die Anlage wie auch für die Outputströme erhoben. Die prognostizierte Zusammensetzung des Anlagenoutputs wurde mit den tatsächlichen Outputströmen verglichen.

Die Verwertungskette zeigt, dass bei derzeitigen österreichischen Sammelmengen und stofflichem Recycling am Stand der Technik 28 bis $31 \%$ der in Verkehr gebrachten Gerätematerialien zurückgewonnen werden können. Anders ausgedrückt bedeutet dies, dass 69 bis $72 \%$ des Materials der in Verkehr gesetzten EKG trotz Sammlung und Recycling verloren gehen. Die größten Materialverluste (und damit einherge-

DI Dr. N. Unger $(\bowtie) \cdot$ DI Mag. P. Beigl • Univ.-Prof. DI Dr. S. Salhofer Department Wasser - Atmosphäre Umwelt, Institut für Abfallwirtschaft, Universität für Bodenkultur Wien, Muthgasse 107, 1190 Wien, Österreich nicole.unger@boku.ac.at

\section{Small waste electrical and electronic equipment - from collection to secondary resource}

Abstract Waste electrical and electronic equipment is one of the fastest growing waste streams in Europe with an annual increase of 3-5\%. Goals and visions of the European Union aim at the prevention of electrical and electronic waste, sustainable resource management and a restriction of hazardous materials. The research presented in this paper, funded by the FFG, evaluates the material flows of small waste and electronic equipment along the full end-of-life process chain and associated material losses, from collection of the devices to recovered secondary resources. Key parts of the research were a review of novel approaches of collection schemes for small devices from households, and the application of a newly developed stochastic model to estimate the material composition of outputs from a pre-treatment facility based on the facility input (collected devices). In close cooperation with the operator of an Austrian pre-treatment facility batch trials were run and primary data collected. Results for the expected output from the facility generated by the stochastic tool were then compared to the actual composition.

The end-of-life process chain indicates that - when taking into account the Austrian collection rates and stateof-the-art material recycling technology
$-28-31 \%$ of the material from small devices put on the market are recovered. In other words, $69-72 \%$ of the materials are lost. The highest losses (and thus the highest improvement potential) occur due to uncollected devices (41\% losses) and in material recycling (30-36\% losses). The review of collection schemes indicates that retail takeback systems and the collection in public spaces are promising approaches to contribute to a higher collection rate. The estimation of the output composition using the stochastic model provides a satisfactory accuracy for printed circuit boards, ferrous metals and plastics.

\section{Einleitung}

Elektrische und elektronische Geräte sind heutzutage aus dem täglichen Leben nicht mehr wegzudenken. Im Beruf, im Haushalt oder in der Freizeit spielen diese Geräte eine immer wichtigere Rolle. Gleichzeitig stellen Elektroaltgeräte (EAG) einen der am schnellsten wachsenden Abfallströme in der EU dar, mit einem jährlichen Zuwachs von 3 bis $5 \%$ (Eurostat 2017). In Österreich werden pro Person jährlich zwischen 18,4 und $20,6 \mathrm{~kg}$ ebensolcher Geräte in Verkehr gebracht (Werte von 2007 bis 2014) (Eurostat 2017). Damit liegt Österreich im europäischen Mittelfeld. Spitzenreiter sind Malta und Norwegen mit 40 bzw. $35 \mathrm{~kg} /$ EW.a. Insgesamt wurden 172.680 Tonnen Elektro- und Elektronikgeräte in 2015 in Österreich in Verkehr gebracht. Im selben Jahr wurden 9,2 kg EAG pro Einwohner gesammelt, was etwa $46 \%$ der in Verkehr gebrachten Geräte entspricht. Elektrokleingeräte (EKG) machen davon $3,57 \mathrm{~kg}$ aus, was $64 \%$ der in diesem Jahr in Verkehr gebrachten EKG entspricht (EAK 2016). Die in Verkehr gebrachten Mengen und Sammelmengen für alle Elektroaltgerätegruppen sind in Abb. 1 dargestellt. Ähnlich ist die Situation in Deutschland, wo im Jahr 2013 in Summe 1.609.232 Tonnen Geräte in 


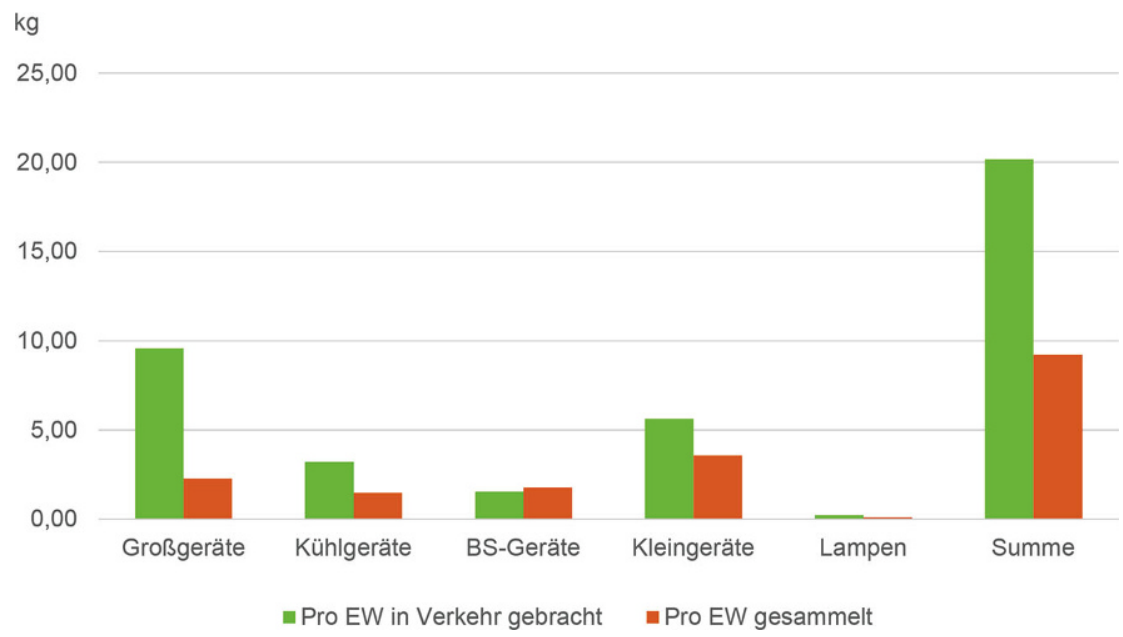

Abb. 1 In Verkehr gebrachte und gesammelte Gerätemengen in Österreich nach Elektroaltgerätegruppen in kg/EW.a (EAK2016)

Verkehr gebracht und $45 \%$ dieser Mengen verwertet wurden (Umweltbundesamt 2017a). Im europäischen Vergleich werden in Norwegen die größten Mengen an EAG pro Einwohner gesammelt (20,9 kg/EW.a) (Eurostat 2017).

Im Vergleich $\mathrm{zu}$ vielen anderen $\mathrm{Ab}$ fallströmen stechen EAG durch ihren hohen Grad an Komplexität und Heterogenität hervor. Sie bestehen großteils aus komplex verbauten Metallen und Kunststoffen und können je nach Gerätetyp gefährliche Bauteile und Substanzen wie z. B. Kondensatoren, Tonerkartuschen, Batterien und Kunststoffe mit bromierten Flammschutzmitteln enthalten (Oguchi et al. 2013; Salhofer und Tesar 2011). Diese Schadstoffe sind entsprechend den Vorgaben der Europäischen Elektroaltgeräterichtlinie unbedingt aus den getrennt gesammelten EAG zu entfernen, um einen Eintrag in den zu verwertenden Abfallstrom sowie in die erzeugten Rezyklate zu verhindern (Europäisches Parlament 2012).

EAG beinhalten jedoch auch eine Reihe an wertvollen Ressourcen wie etwa Kupfer, Aluminium, Gold, Silber und Kunststoffe (Huisman et al. 2007; Geyer und Blass 2010; Oguchi et al. 2011). Da die Entscheidung, welche Materialen in welchem Ausmaß rückgewonnen werden, nicht nur aufgrund technischer, sondern auch wirtschaftlicher Überlegungen getroffen wird, wird derzeit nur ein Teil dieser Ressourcen rückgewonnen und der Wirtschaft als Sekundärmaterial wieder zur Verfügung gestellt (vgl. Chancerel et al. 2009).

Im 2015 veröffentlichten Kreislaufwirtschaftspaket (Europäische Kom- mission 2015) sind fünf sektorale Schwerpunktbereiche festgelegt. Diese bezeichnen Wirtschaftssektoren, die im Zusammenhang mit der Kreislaufwirtschaft aufgrund der Besonderheiten ihrer Produkte oder Wertschöpfungsketten, ihres ökologischen Fußabdrucks oder ihrer Abhängigkeit von Materialien aus Ländern außerhalb der EU mit besonderen Problemen konfrontiert sind. Zwei dieser fünf Schwerpunktbereiche sind besonders für EAG relevant, nämlich „Kunststoffe“ sowie „kritische Rohstoffe" $^{\text {"l }}$. Speziell wird hervorgehoben, dass nur hochwertiges Recycling die Verwertung von kritischen Rohstoffen gewährleisten kann, wobei die Sammlung, die Demontage und das Recycling von Produkten dabei von entscheidender Bedeutung sind. Für die Ressourcenrückgewinnung sind vor allem Elektrokleingeräte interessant, da sie eine höhere Wertstoffdichte aufweisen als beispielsweise weiße Ware. Nicht nur aus Sicht der Ressourceneffizienz stellen sich daher die Fragen, wie gut die Rückgewinnung von Ressourcen derzeit funktioniert, wo Materialien verloren gehen und in welchen Stufen der Verwertungskette derzeit ungenutzte Verbesserungspotenziale bestehen.

Im Rahmen eines FFG-Forschungsprojekts, an dem die Montanuniversität Leoben, die Universität für Bodenkultur Wien sowie die Müller-Guttenbrunn Gruppe beteiligt waren, wurden

${ }^{1}$ Kritische Rohstoffe sind Rohstoffe, die in der EU von großer wirtschaftlicher Bedeutung sind und für die zugleich ein hohes Risiko für Versorgungsengpässe besteht. die Stoffströme von Elektrokleingeräten in der mechanischen Aufbereitung eines österreichischen Behandlungsbetriebs sowie entlang der gesamten Verwertungskette von der Sammlung bis hin zur Sekundärressource untersucht. Dabei wurden neue Ansätze zur Sammlung von Elektrokleingeräten aus Haushalten identifiziert und die damit erreichten Sammelmengen dargestellt. Dafür wurden die bestehende Literatur und Projektberichte gesichtet sowie Praxiserfahrungen mittels Experteninterviews zusammengetragen.

In der Vergangenheit wurde die Steuerung und Optimierung des Betriebs von Aufbereitungsanlagen erfahrungsbasiert und ausschließlich durch outputseitige Beprobung und Beurteilung der Materialströme vorgenommen. Damit die Konfiguration der mechanischen Aggregate besser abgestimmt werden kann, ist eine A-prioriAbschätzung der Zusammensetzung des Anlagenoutputs nützlich. Dazu wurde ein neues, stochastisches Modell zur Abschätzung der Materialzusammensetzung des Outputs entwickelt. Im Rahmen von Batchversuchen wurde als Grundlage dafür das Inputmaterial der mechanischen Aufbereitungsanlage auf Basis von Zerlegungsdaten und Gerätezählungen charakterisiert und der Anlagenoutput abgeschätzt. Dieser wurde mit den tatsächlichen, vor Ort mittels Handklaubungen beprobten Outputströmen verglichen.

\section{Getrennte Sammlung von Elektrokleingeräten}

Die Sammlung ist der erste wichtige Schritt in der Verwertungskette von EAG und Grundvoraussetzung für eine hochwertige Rückgewinnung von Ressourcen (Europäische Kommission 2015). Das Vorhandensein eines Sammelsystems zur getrennten Erfassung ist eine Grundvoraussetzung für das Sammeln von EAG. Es garantiert allerdings nicht, dass alle EAG über dieses System entsorgt werden. Gerade EKG können aufgrund ihrer geringen Größe leicht im Haushalt oder Betrieb gelagert oder anders entsorgt werden (z. B. über den Restmüll) und gehen somit für die Rückgewinnung verloren.

In der neuen Europäischen Elektroaltgeräterichtlinie (Europäisches Parlament 2012), umgesetzt in der Novelle 2014 zur österreichischen Elektroaltgeräteverordnung (BKA 2014), wurden ambitionierte Vorgaben bezüglich der 


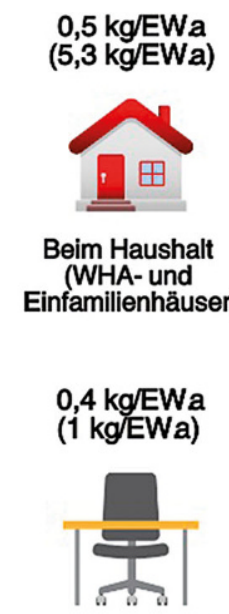

Sammlung am Arbeitsplatz
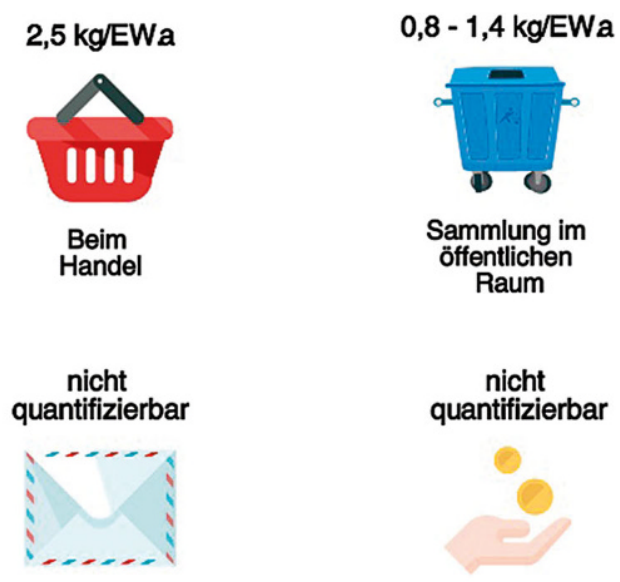

Sammlung per Post nicht quantifizierbar

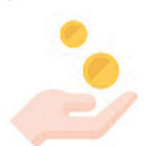

Pfandsystem

Abb. 2 Elektrokleingeräte-Sammelschienen und zu erwartende Sammelmengen, die Werte in Klammer sind berichtete Höchstwerte (WHA Wohnhausanlagen). Die Graphik wurde in easelly erstellt

Sammelquoten festgeschrieben, die deutlich über das bisherige, in Österreich leicht erreichbare Sammelziel von $4 \mathrm{~kg} /$ EW.a hinausgehen. Die Mindestsammelquote beträgt demnach ab 2016 $45 \%$ des durchschnittlichen Marktinputs der letzten 3 Jahre. Diese Quote wird ab 2019 weiter auf $65 \%$ des durchschnittlichen Marktinputs der letzten 3 Jahre oder auf $85 \%$ des Aufkommens von EAG erhöht. Während die seit 2016 gültigen Zielvorgaben wahrscheinlich knapp erfüllt werden können, werden jene, die ab 2019 gültig sind, nach jetziger Einschätzung wahrscheinlich nicht erreicht werden können.

Das Ziel der Recherche von bestehenden EKG-Sammelsystemen war es, neue Ansätze zur Sammlung von EKG aus Haushalten darzustellen, die über die bisher in Österreich praktizierte Sammlung bei Altstoffsammelzentren (oder vergleichbaren Einrichtungen) hinausgehen und somit Beiträge zur Erhöhung der EKG-Sammelmengen liefern könnten. Die Recherche basierte auf Auswertungen von Pilotversuchen und Studien in Europa sowie begleitenden Experteninterviews. Die auf diese Weise recherchierten Werte (zumeist Sammelerfolg pro Einwohner und Jahr) müssen jedoch mit Vorsicht betrachtet werden. Die Gründe dafür sind:

- Eingeschränkte Übertragbarkeit der Ergebnisse aus Pilotversuchen auf den Langzeitbetrieb.

- Keine Berücksichtigung von Verschiebungen zwischen Sammelschienen.

- Fehler bei der Zuordnung nach Gerätekategorien im Rahmen der Dokumentation von Sammelmengen.

- Uneinheitliche Vorgaben, welche Gerätetypen in Sammelschienen erfasst werden sollen.

- Miterfassung von anderen Typen von Elektroaltgeräten (Fehlwürfe), z. B. $26 \%$ Elektrogroßgeräte in der EKG-Sammlung in Kopenhagen (Kiil 2017).

- Potenzieller, illegaler Export von EKG durch informell tätige Sammler.

Ein Überblick über die unterschiedlichen Sammelschienen und die jeweils erreichten Sammelmengen ist in Abb. 2 zu sehen. Es hat sich gezeigt, dass die gesammelten Mengen einzelner Sammelschienen im Allgemeinen gering und teilweise nicht quantifizierbar sind. Relevante und aussagekräftige Beispiele für bestehende Sammelschienen und Pilotversuche in Europa werden in den folgenden Abschnitten kurz umrissen.

\subsection{Sammlung beim Haushalt}

Bei der Sammlung beim Haushalt lässt sich zwischen Sammlung in Wohnhausanlagen (WHA) und Sammlung bei Einfamilienhäusern unterscheiden. Es wurden Studien und Artikel zu 13 Systemen (zumeist Pilotversuche) in europäischen Städten untersucht. Die berichteten Sammelmengen liegen zwischen 0,02 und 5,32 kg/EW.a, wobei der Maximalwert wenig aussagekräftig ist, da er neben EKG auch andere gesammelte EAG sowie Batterien, Glüh- birnen und Kompaktleuchtstoffröhren beinhaltet. Ein aussagekräftiges Beispiel sind die Werte aus Kopenhagen, das ein etabliertes Sammelsystem für EKG beim Haushalt hat, welches bis vor Kurzem sowohl bei Einfamilienhäusern, als auch in Wohnhausanlagen verwendet wurde. Dabei erhält jeder Haushalt eine Sammelkiste (Red Box), die in gewissen Abständen im Holsystem geleert wird. Inzwischen wurde die Sammlung in Wohnhausanlagen jedoch flächendeckend umgestellt und die Red Box durch eine zentrale EKG-Sammlung in den Wohnhausanlagen ersetzt (Kannegaard 2015; City of Copenhagen 2017). Dies ist daher nur eine Änderung der Sammlung, nicht eine Neueinführung. Die in Kopenhagen erreichten Sammelmengen für EKG beim Haushalt lagen 2016 bei 0,38 kg/EW.a (Kiil 2017). Die Kosten vor Steuern für dieses Sammelsystem belaufen sich auf etwa $9,89 €$ pro Haushalt (Tomasin 2013). In Hamburg wurde ein Konzept getestet, bei dem Kunden, die an eine Sammlung der Altpapiertonne angeschlossen sind, ein Wertstofftag für die Entsorgung von EAG, Altkleidern und Schuhen angeboten wurde. Dabei wurden unterschiedliche Varianten der Sammlung getestet (z.B. Nutzung der Altpapiertonne am Tag nach der Entleerung für Wertstoffsammlung; Beistellen eines Sackes am Tag der Altpapiersammlung; Aktionstage in WHA mit Infoständen; unbegleitete, temporäre Behälteraufstellung). Obwohl Wertstofftage von den Betreibern grundsätzlich als praktikabel eingeschätzt wurden, waren die Kosten für die Mono-Sammlung sehr hoch und das System wurde vor dem Hintergrund der erzielten Sammelergebnisse als uneffektiv eingeschätzt (Winterberg 2013).

\subsection{Sammlung beim Handel}

Einige europäische Länder haben rechtliche Vorgaben zur Rücknahme von Altgeräten durch den Handel. In Österreich ist der Handel verpflichtet, auf Verlangen des Kunden Geräte bei jedem Neukauf kostenlos zurückzunehmen, sofern das zurückgegebene Gerät dem neu gekauften Gerät gleichwertig ist und dieselbe Funktion erfüllt (Zug-umZug- oder 1:1-Rücknahme). Von dieser Verpflichtung sind nur jene Betriebe ausgenommen, deren Verkaufsfläche weniger als $150 \mathrm{~m}^{2}$ beträgt (BKA 2005).

In Deutschland sind seit 24. Juli 2016 „Vertreiber“ für Elektro- und Elektro- 
nikgeräte mit einer Verkaufsfläche von mindestens $400 \mathrm{~m}^{2}$ dazu verpflichtet, beim Verkauf eines neuen Gerätes ein Altgerät der gleichen Geräteart und mit gleichen Funktionen unentgeltlich zurückzunehmen (1:1-Rücknahme) und Altgeräte, die in keiner äußeren Abmessung größer als 25 Zentimeter sind, in haushaltsüblichen Mengen auch ohne Neukauf unentgeltlich zurückzunehmen (0:1-Rücknahme). Dasselbe gilt auch für den Versandhandel, wobei sich die Mindestfläche von $400 \mathrm{~m}^{2}$ in diesem Fall auf die gesamte Lagerund Versandfläche des Händlers bezieht (Umweltbundesamt 2017b). In der Schweiz schreibt die Verordnung über die Rückgabe, die Rücknahme und die Entsorgung elektrischer und elektronischer Geräte (VREG) vor, dass der Einzelhandel Geräte jener Arten, die im Sortiment geführt werden, kostenlos vom Endverbraucher zurücknehmen muss (BAFU 2017).

Rücknahmesysteme durch den Handel wurden in sechs Städten untersucht. Die berichteten Sammelmengen reichen von einigen $100 \mathrm{~kg}$ pro Sammelstelle und Jahr bis zu 2,5 kg/EW.a. Das Sammeln von EKG kann direkt beim Handel erfolgen (z. B. Annahme durch einen Mitarbeiter oder in einem Sammelbehälter) oder in dessen unmittelbarer Umgebung (z. B. durch das Aufstellen eines zentralen Sammelbehälters in einem Einkaufszentrum). Die Sammlung kann des Weiteren mit einem karitativen Zweck oder anderen Anreizen für Kunden wie beispielsweise der Ausgabe von Gutscheinen beim Binee System (RepaNet 2017) verbunden sein. Besonders hohe Sammelmengen werden in Norwegen erreicht. In Stavanger beispielsweise werden zwei Drittel (2,5 kg/EW.a) der insgesamt gesammelten EKG aus Haushalten (3,7 kg/EW.a) über den Handel zurückgenommen (Meissner 2017). Dies wird durch die seit langem in Norwegen etablierte, landesweite und freiwillige Rücknahme des Handels begünstigt. Insgesamt wurden in Stavanger im Jahr 2016 etwa 17,9 kg/EW.a EAG gesammelt (Larsen 2017). Dabei ist jedoch zu berücksichtigen, dass Norwegen auch den zweithöchsten Konsum an elektrischen und elektronischen Geräten in Europa hat.

\subsection{Sammlung im öffentlichen Raum}

Sammelbehälter für EKG können bei Sammelinseln oder an hoch frequentierten Plätzen aufgestellt sein. Häufig werden Sammelbehälter (z. B. 240 l, 11001, Depotcontainer) verwendet. Da diese Behälter uneingeschränkt zugänglich sind, berichten einige Systembetreiber von der Entnahme der Sammelware durch Unbefugte und - damit einhergehend - von Beschädigung der Behälter, Verschmutzung rund um die Behälter und Fehlwürfen. Im Rahmen der hier vorgestellten Untersuchung wurden Berichte zu zehn Sammelsystemen im öffentlichen Raum (die meisten davon aus Deutschland) analysiert. Die berichteten Sammelmengen liegen zumeist zwischen 0,04 und 1,5 kg/EW.a, teilweise sind jedoch nur Gesamtmengen pro Behälter und Jahr angegeben. Laut einer Studie des bifa Umweltinstituts von Nordsieck et al. (2016) können ab einer Containerdichte von mehr als 0,2 Depotcontainer pro 1000 Einwohner etwa 0,8 bis $1,8 \mathrm{~kg} / \mathrm{EW}$.a Sammelgut erzielt werden.

Die hier angeführten Sammelmengen beziehen sich auf die Zeit vor der Einführung der ADR/RID-2015-Richtlinie (United Nations 2015), wonach sämtliche lithiumhaltigen Batterien und Zellen im internationalen Transportrecht als Gefahrengut eingestuft werden. Seit deren Inkrafttreten besteht große Ungewissheit bezüglich der Gesetzeskonformität der Verwendung von Depotcontainern in der EKGSammlung. Mehrere Kommunen reduzieren oder stoppen diese daher. Das Gefahrengutrecht verbietet den Transport von Elektroaltgeräten mit LithiumBatterien als Primärquelle sowie von losen Lithium-Batterien in loser Schüttung. Den Experten des VKU (VKU 2017) zufolge erweist sich die Verwendung von Umleercontainern als schwer umsetzbar, da Fehlwürfe in Form von losen Lithium-Batterien in der Praxis nur schwer auszuschließen sind. Als ADR-konforme Alternative ist unter gewissen Voraussetzungen die Verwendung von Wechselcontainern zulässig, wobei sich aufgrund des logistischen Mehraufwands höhere Kosten ergeben. Das Gefahrenpotenzial der Verwendung von Depotcontainern wurde von Hobohm et al. (2016) sowie Nordsieck et al. (2016) untersucht. Beide Studien zeigen, dass das Gefährdungspotenzial durch Lithium-Batterien als sehr gering einzuschätzen ist.
2.4 Andere Sammelschienen und unterstützende Maßnahmen

Ein bereits öfter umgesetzter Ansatz ist das Sammeln von EKG über den Postweg. Die Abwicklung kann über im Haushalt ausdruckbare Versandmarken z. B. beim „Electroreturn“-System (Deutsche Post 2017) sowie durch Bereitstellung von speziellen Kuverts oder anderen Sammelbehältnissen erfolgen. Diese Art der Sammlung lässt sich gut mit einem karitativen Zweck verbinden. Die Ö3-Wundertüte ist eines der erfolgreichsten Systeme dieser Art mit jährlich zwischen 310.000 und 470.000 gesammelten Mobiltelefonen, wobei sich der Mittelwert bei knapp 400.000 Mobiltelefonen pro Jahr bewegt (Beigl et al. 2012).

Die Sammlung von EKG am Arbeitsplatz wurde in einem deutschen Pilotversuch erprobt, wobei Sammelmengen von etwa $0,4 \mathrm{~kg} /$ Mitarbeiter.a erreicht wurden. Die Spitzenwerte reichten bis zu 1 kg/Mitarbeiter.a (Winkel, s.a).

Neben den zuvor beschriebenen Sammelschienen gibt es auch eine Reihe unterstützender Maßnahmen, die Motivation und Hilfestellung bei der Sammlung von EKG geben sollen. In einigen Kommunen werden Sammelbehälter wie Karton- oder Kunststoffschachteln, Säcke und Ähnliches zum Vorsammeln im Haushalt zur Verfügung gestellt. Beispiele dafür sind u.a. die Wetterauer E-Box (Abfallwirtschaftsbetrieb Wetterau 2017) oder die VorSammeltasche Salzburg (Stadt Salzburg 2017).

Informationskampagnen und Öffentlichkeitsarbeit sind für alle Sammelschienen wichtig, können jedoch kostspielig sein. In Deutschland gibt es seit einiger Zeit eine E-Schrott-App. Dabei handelt es sich um eine kostenlose Applikation, mit der Haushalte die Öffnungszeiten der kommunalen und privaten Sammelstellen abrufen und illegal entsorgte EAG unkompliziert melden können sowie weitere Informationen bekommen (BMUB 2017). Die Einführung eines Pfandsystems für Mobiltelefone wird von Trischler et al. (2015) vorgeschlagen.

\section{Aufbereitung und Recycling}

Auf die Sammlung folgen als nächste Schritte in der Verwertungskette die Aufbereitung und die Verwertung. Die Aufbereitung von Elektroaltgeräten erfolgt typischerweise in drei 


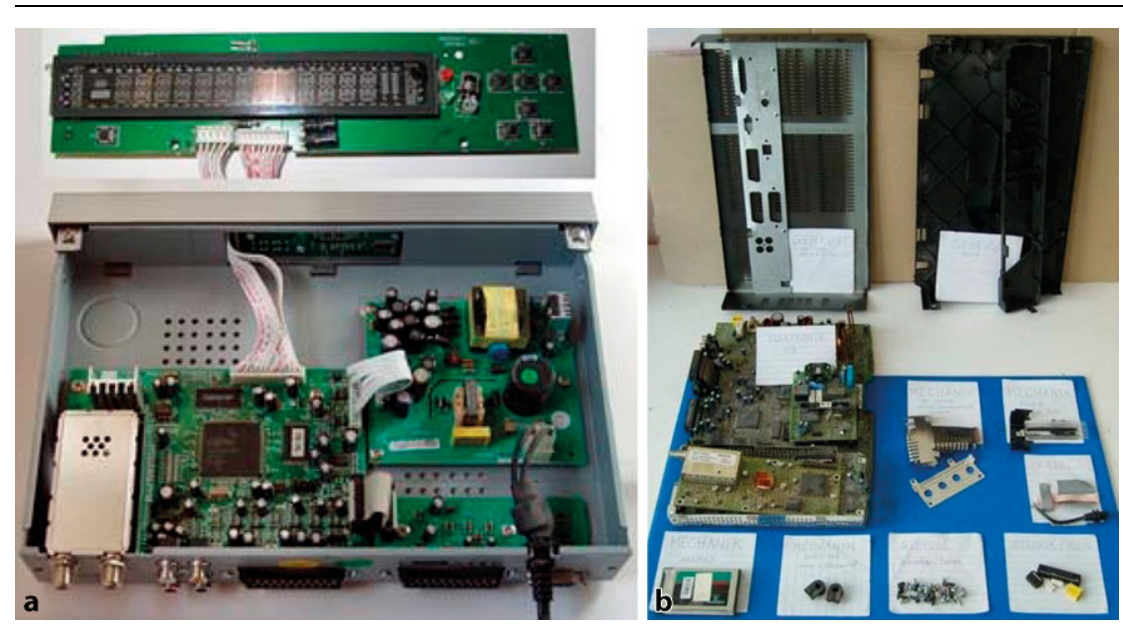

Abb. 3a,b Gerätedemontage am Beispiel eines Receivers

Schritten: Bei der Demontage werden schadstoffhaltige Bauteile entsprechend den Vorgaben der EAG-Richtlinie (Europäisches Parlament 2012) bzw. für Österreich der Abfallbehandlungspflichtenverordnung (BKA 2004) abgetrennt, häufig werden im selben Schritt wertstoffreiche Bauteile (z. B. Kabel) entnommen. Die Demontage erfolgt teilweise manuell (Aufschrauben oder Aufbrechen der Gehäuse, Entnahme der Zielbauteile) oder mittels mechanischer Unterstützung zum Aufbrechen der Geräte und anschließender Entnahme der Zielbauteile auf einem Sortierband. Bei der nachfolgenden mechanischen Aufbereitung kommen Aggregate zum Einsatz, die die komplexen Materialverbunde zerkleinern und trennen, womit die Hauptstoffgruppen, wie z. B. Eisen und Stahl, Nichteisenmetalle und Kunststoffe voneinander getrennt werden. Weitere Trennschritte dienen der genaueren Auftrennung der Materialien. Die Outputströme dieser Aufbereitung werden in weiterer Folge den stofflichen Verwertungsprozessen zugeführt, beispielsweise durch Einsatz von Stahlschrott in der Stahlindustrie. Stofflich nicht verwertbare Materialien werden in Österreich überwiegend in Müllverbrennungsanlagen thermisch verwertet. Die bei der Demontage anfallenden, schadstoffhaltigen Bauteile müssen ebenfalls einer geeigneten Behandlung zugeführt werden.

In Ländern wie Österreich oder Deutschland sind in den letzten 20 Jahren zahlreiche Aufbereitungsanlagen entwickelt und gebaut worden, die unterschiedliche Technologien und verschiedene Kombinationen von Aggregaten einsetzen, um die Aufschluss- und Trennprozesse zu optimieren. Typische Aggregate sind Shredder (Autoshredder oder spezifische Mittelshredder) zur Fragmentierung, Windscheider, Magnetabscheider, Wirbelstromscheider, Sink-Schwimmanlagen und andere Aggregate zur Trennung der Materialströme. Damit können Inputs wie gemischte Elektrokleingeräte relativ gut aufgeschlossen und in vermarktbare Fraktionen (Schrotte, Kunststoffe) übergeführt werden. Für das Ausmaß der erzielbaren Rückgewinnungsquoten ist die betriebsinterne Wahl der Verwertungsroute entscheidend, die von der Kompatibilität des Materialstroms mit der angewendeten Technologie und der Möglichkeit zur Gewinnung von Sekundärprodukten abhängt. Eine ungünstige Abstimmung von Outputcharakteristika und Verwertungsroute würde zum Verlust der darin enthaltenen Wertstoffe führen. Bei komplexen Materialströmen ist insbesondere die Abwägung von Kupfer- und Edelmetallgehalten im Vergleich zu massenrelevanten Metallen (v. a. Eisen) zu treffen. Bei der Kupferroute beispielsweise werden wertvolle Komponenten bereits ab einem Kupfergehalt von $5 \%$ zugeführt, da Kupfer ein wertvoller Rohstoff ist und das enthaltene Eisen zur Schlackenbildung benötigt wird. Neben der technischen ist auch eine betriebswirtschaftliche Abstimmung der Outputströme auf die darauffolgende Verwertung wichtig, um beim Überschreiten von Schwellenwerten die finanzielle Kompensation von enthaltenen Wertstoffen zu gewährleisten, da bei bestimmten Verwertungsrouten nicht alle Wertstoffe finanziell abgegolten werden.
In der Vergangenheit wurde die Steuerung und Optimierung des Betriebs von Aufbereitungsanlagen im Wesentlichen erfahrungsbasiert und durch Beurteilung der Outputströme vorgenommen, wobei aufgrund der starken Heterogenität von Elektroaltgeräten stets von einer gleichbleibenden Zusammensetzung des Inputs ausgegangen wurde (Beigl und Salhofer 2016). Bezüglich Heterogenität nehmen EKG in der Abfallwirtschaft eine Sonderrolle ein, da dieser Abfallstrom betreffend Stückmasse (z.B. Computermaus vs. Mikrowellenherd), Bauteilkomplexität (z. B. Multifunktionsdrucker) und Wertstoffgehalt (z.B. Kaffeemaschine vs. Receiver) gegenüber anderen Abfallarten besonders deutliche Variation aufweist und damit die Abschätzung von Metall- oder Wertstoffgehalten erschwert.

An der Universität für Bodenkultur Wien wurde ein neuer Ansatz zur Charakterisierung von EKG entwickelt, der eine Prognose der Zusammensetzung des Outputs der Aufbereitungsanlagen auf Basis der Beurteilung des Inputmaterials (Sammelware) ermöglicht. Das entwickelte stochastische Modell baut auf einer breiten Datenbasis und einer detaillierten Klassifikation nach 216 Gerätetypen und nach 123 Materialien, d. s. Monomaterialien $(\mathrm{Fe}, \mathrm{Cu}$, Kunststoffe), Verbunde (z. B. Kabel) und komplexe Bauteile (z. B. Motoren, Lüfter, Laufwerke) auf (Beigl und Salhofer 2016). Als Grundstein für die Datenbasis wurden umfangreiche Zerlegeversuche durchgeführt, um Daten über die Materialzusammensetzung von relevanten Gerätearten (beispielsweise PCs, Staubsauger etc.) zu gewinnen (Abb. 3). Derzeit liegen über 1100 Datensätze dazu vor. Zusätzlich zu gerätespezifischen Zerlegungen wurde die Materialzusammensetzung von komplexen Bauteilen wie Festplatten, CD/DVD-Laufwerken und Elektromotoren separat analysiert.

Im Rahmen dieses Artikels wird auf die mechanische Aufbereitung von 315 Tonnen an Elektrokleingeräten aus österreichischen Haushalten näher eingegangen, die im Zeitraum der Jahre 2015 und 2016 in drei Batchversuchen (jeweils mit ca. 100 Tonnen) verarbeitet wurden. Die Datengrundlage umfasst die erhobene Masse und Zusammensetzung von Input- und allen Outputflüssen. Der Input wurde mittels Gerätezählungen, die für jeden Batch durchgeführt wurden (ca. 4 Tonnen pro Batch), charakterisiert und die 


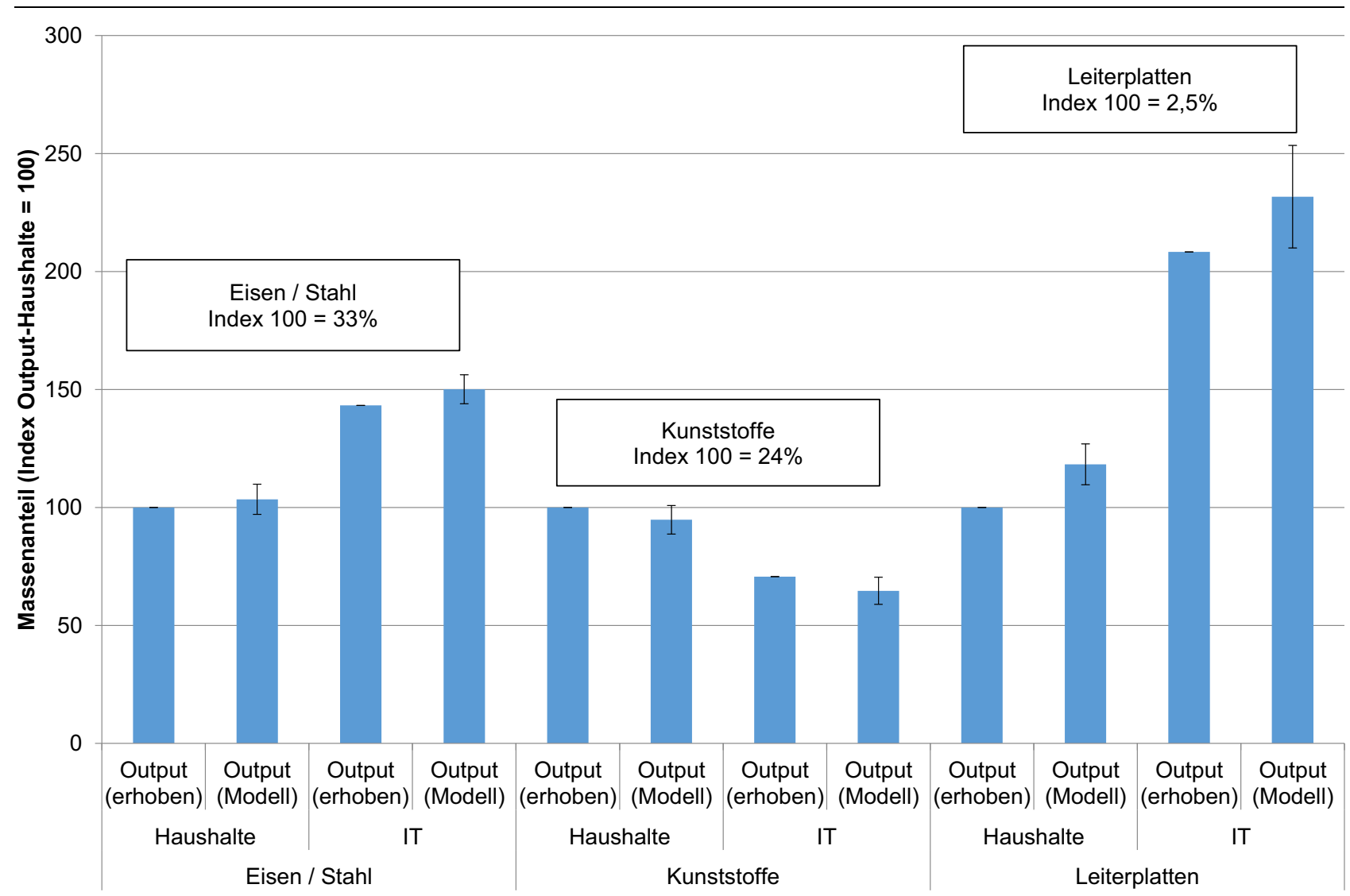

Abb. 4 Prognostizierte und erhobene Massenanteile im Output für Eisen/Stahl, Kunststoffe und Leiterplatten bezogen auf den jeweiligen Output der EKG-Aufbereitung aus Haushalten

Zusammensetzung der Sammelware nach Gerätearten bestimmt. Anschließend wurde das Material aufbereitet und die Outputströme wurden ebenfalls einer detaillierteren Analyse (im Wesentlichen Sortieranalyse) zugeführt. Outputseitige Daten des gesamten Prozesses umfassen Ergebnisse von Handklaubungen und pyrometallurgischen Analysen zu über 50 Outputströmen sowie deren Verwertung. Verwertungsrouten umfassen das Materialrecycling für Kupferroute (inkl. anschließender Edelmetallschmelze) zur Gewinnung von Kupfer, Blei, Nickel, Zink, Gold, Silber und Palladium, Eisen- und Stahlrecycling, Aluminiumrecycling, Kunststoffrecycling zur Rückgewinnung von Polystyrol, Polypropylen und ABS sowie Batterienrecycling. Zusätzlich wird Energie aus der thermischen Verwertung gewonnen. Ein kleiner Anteil des Outputs wird deponiert.

Die Auswertung der in der mechanischen Aufbereitung erhobenen Daten hat gezeigt, dass mit 41,0\% der Großteil in EKG enthaltenen Materialien ins Eisen- und Stahlrecycling geht. Weitere
$21,3 \%$ gehen ins Kunststoffrecycling, $19,3 \%$ in die thermische Verwertung, $13,5 \%$ in die Kupferverwertungsroute (inkl. Edelmetallschmelze), 4,3\% ins Aluminiumrecycling und 0,3\% ins Batterienrecycling. 0,2 \% der in EKG enthaltenen Materialien werden deponiert.

Für einzelne Materialströme, wie Eisen und Stahl, Kunststoffe sowie Leiterplatten wurden mithilfe des stochastischen Modells die modellbasierte Prognose des Outputs mit der tatsächlichen, vor Ort erhobenen Outputzusammensetzung validiert. Das erlaubt die Abschätzung des Anlagenoutputs auf Basis der Zusammensetzung der Sammelware (d.h. Mix nach Gerätetypen) sowie der Gerätezusammensetzung. Beispielsweise wurde bei IT-Sammelware ein Leiterplattengehalt von $5,7 \%$ prognostiziert und 5,2\% im Output wiedergefunden. Bei EKG aus Haushalten wurde ein Leiterplattenanteil von 2,9\% prognostiziert bzw. 2,5\% erhoben. Bei Eisen/Stahl sowie Kunststoffen wurden ebenfalls Ergebnisse mit zufriedenstellender Genauigkeit erzielt. Abb. 4 zeigt die Relationen von erhobenen und pro- gnostizierten, modellbasierten Materialanteilen des Anlagenoutputs, jeweils bezogen auf den tatsächlichen Materialanteil in EKG aus Haushalten (Index = 100).

Dieser Abgleich ist allerdings noch nicht für alle Fraktionen möglich, insbesondere Materialien, die überwiegend im Verbund in komplexen Bauteilen eingesetzt sind (wie $\mathrm{Al} / \mathrm{Mg}$ ) erfordern noch detailliertere Untersuchungen.

\section{Materialkaskade}

Den Ausgangspunkt zur Abbildung der gesamten Verwertungskette für EKG - von der Sammlung bis zur Sekundärressource - in Form einer Materialkaskade bildet die Menge an in Verkehr gebrachten Geräten. Da anzunehmen ist, dass ein Großteil der Geräte nicht bereits im Jahr nach Inverkehrbringen entsorgt wird, wird in Anlehnung an die neue EU-Altgeräterichtlinie der durchschnittliche Marktinput der letzten 3 Jahre als Annäherung an die in Verkehr gebrachten Mengen herange- 


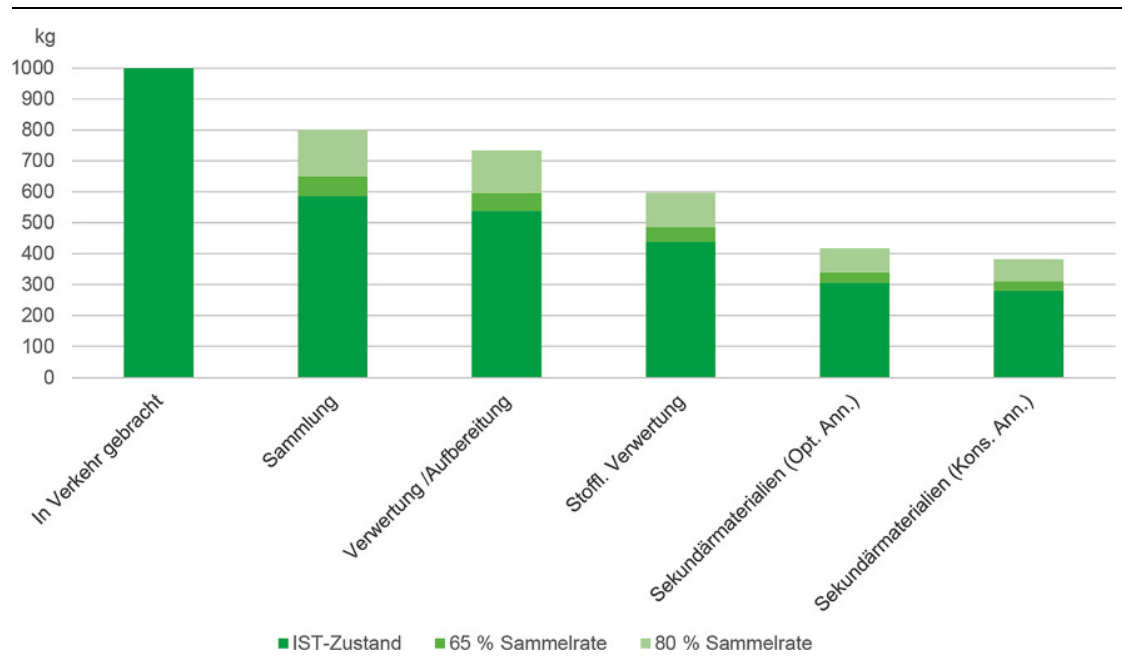

Abb. 5 Materialkaskaden des IST-Zustandes sowie bei Erhöhung der Sammelrate auf $65 \%$ bzw. $80 \%$

zogen. Im Rahmen der in diesem Artikel präsentierten Studie wurden die österreichischen Verwertungsketten der EAG Gerätekategorien 2 bis 9 (Annex I, II in: Europäisches Parlament 2012) abgebildet. In den Jahren 2011 bis 2013 wurden im Schnitt 77.700 t dieser Geräte in Österreich in Verkehr gebracht. Die entsprechende Sammelmenge für 2014 lag bei $45.600 \mathrm{t}$ bzw. 58,66\%. Ein geringer Teil der gesammelten Menge (811 t) wurden der Wiederverwendung zugeführt, $41.830 \mathrm{t}$ der thermischen oder stofflichen Verwertung. Entsprechend den aktuellen Daten von Eurostat (2017) liegt die stoffliche Verwertungsquote im europäischen Durchschnitt bei $81,30 \%$. Dies deckt sich sehr gut mit den beim Verwertungsbetrieb erhobenen Verwertungsquoten $(80,5 \%)$.

Zur Detaillierung der Materialkaskade wurden Primärdaten aus den Batchversuchen in der mechanischen Aufbereitung verwendet. Die Kenntnis der genauen Zusammensetzung sowie des weiteren Verwertungswegs für jeden Outputstrom der Aufbereitungsanlage erlaubt die Bestimmung des Anteils der recyclingrelevanten Materialien in jeder Verwertungsroute, also Materialien, die in der jeweiligen Verwertungsroute potenziell rückgewonnen werden können. Weiterführend lässt sich daraus abschätzen, welcher Anteil dieses recyclingrelevanten Materials auch tatsächlich in jeder Verwertungsroute rückgewonnen wird. Für die Metallrückgewinnung wurden zwei unterschiedliche Annahmen getroffen: Die optimistische Annahme geht davon aus, dass die Ge- samtheit der rückgewinnbaren Metalle als Sekundärmaterial rückgewonnen wird. Bei der konservativen Annahme ist dies nur für $90 \%$ der im Recyclingstrom enthaltenen Metalle möglich. Die tatsächliche Rückgewinnungsrate für Kunststoffe ist bekannt und beträgt $47 \%$. Details $\mathrm{zu}$ den Trennschritten und Beschreibungen der Outputströme und Rückgewinnungsraten sind in Unger et al. (2017) und Höggerl (2015) nachzulesen.

Die Abbildung der gesamten Materialkaskade ergibt sich durch Konsolidierung der Informationen über die einzelnen Schritte der Verwertungskette mit der in Verkehr gebrachten Menge. Abb. 5 zeigt die Materialkaskaden des österreichischen IST-Zustands bezogen auf $1000 \mathrm{~kg}$ EKG, die 2011 bis 2013 in Österreich in Verkehr gebracht wurden. Die Sammelquote beträgt $58,66 \%$, die Recyclingquote $81,30 \%$ (bezogen auf den Anteil der Sammelmenge, der einer Verwertung zugeführt wird). Die Menge an derzeit (IST-Zustand) rückgewonnenem Sekundärmaterial liegt (je nach Annahme, konservativ oder optimistisch) zwischen $28 \%$ und $31 \%$ (kleinste, dunkle Balken). Aufbauend auf diesen Ergebnissen wurde eine theoretische Materialkaskade berechnet, die sich, bei gleicher Technologie, aufgrund einer Sammelquote von $65 \%$ (Vorgabe ab 2019) ergeben würde. Dabei würden 31 bis $34 \%$ des in Verkehr gebrachten Materials rückgewonnen werden. Die höchsten, hellen Balken entsprechen der theoretischen Materialkaskade, die bei einer Sammelquote von $80 \%$ erreicht würden. In diesem Fall würden zwischen $38 \%$ und $42 \%$ des in Verkehr gebrachten Materials rückgewonnen werden.

Die Materialkaskade zeigt zwei bedeutsame Sprünge, die auf große Materialverluste hinweisen: Die größten Materialverluste treten bei allen Szenarien in der Sammlung auf, wo im ISTZustand 41 \% der Gerätemasse verloren geht. Der zweite große Sprung entsteht aufgrund der begrenzten Materialausbeute in der stofflichen Verwertung, bei der die Verluste derzeit zwischen $30 \%$ und $36 \%$ des rückgewinnbaren Materials betragen. Während Metalle im Allgemeinen gut rückgewonnen werden können, liegt die Ausbeute bei Kunststoffen, selbst bei einem Recycling in einer Anlage mit höchstem technischen Standard, bei unter $50 \%$. Die nicht rückgewonnenen Kunststoffanteile werden energetisch genutzt und können auf diese Weise Energieträger ersetzen. Auf die Auswirkungen dieser Rohstoffsubstitution wird in diesem Artikel jedoch nicht näher eingegangen.

\section{Schlussfolgerungen}

Dieser Artikel befasst sich mit der Verwertungskette von Elektrokleingeräten. Die für die Sammlung und die mechanische Aufbereitung dargestellte Materialkaskade quantifiziert die Effizienz der Materialrückgewinnung der in Verkehr gebrachten Produkte, wenn sie einer getrennten Sammlung und anschießenden Verwertung zugeführt werden. Sie veranschaulicht, dass durch stoffliches Recycling ein Teil der in Verkehr eingesetzten Materialien zurückgewonnen werden kann, jedoch die derzeit erzielten Rückgewinnungsmengen von 28 bis $31 \%$ der in Verkehr gebrachten Geräte wahrscheinlich nicht den Erwartungen der Gesellschaft entsprechen.

Anders ausgedrückt gehen in Österreich trotz etablierten Sammelsystems und mechanischer Aufbereitung auf sehr hohem Niveau mit langjährigen Erfahrungen und Know-how 69 bis $72 \%$ des Materials der in Verkehr gesetzten EKG verloren. Ressourcen, die durch die Energiegewinnung aus der thermischen Verwertung eingespart werden, sind hierbei nicht berücksichtigt. Selbst bei Erhöhung der Sammelquote auf $65 \%$ oder $80 \%$ können ,nur' bis $\mathrm{zu} 42 \%$ des Materials rückgewonnen werden. Darüber hinaus muss berücksichtigt werden, dass Sekundärmaterial nicht immer qualitativ gleichwertig mit Primärmaterial ist. Dies kann durch 
Einführung von Qualitätsfaktoren (beispielsweise auf physischer oder ökonomischer Basis) berücksichtigt werden, reduziert damit auch die Menge der durch das stoffliche Recycling am Markt ersetzen Materialien gegenüber den gewonnenen Sekundärmaterialien.

Um die Ausbeute an Sekundärressourcen durch Verwertung von EKG zu verbessern sowie um die ab 2019 gesetzlich vorgeschriebenen Sammelquote von $65 \%$ erfüllen zu können, müssen die Verluste in der Sammlung und in der Verwertung reduziert werden.

Um die Sammelmengen in Österreich zu erhöhen wird vorgeschlagen:

- Bessere Unterbindung von illegalen Exporten.

- Einführung einer 0:1-Rücknahme für den Handel.

- Förderung von freiwilligen Initiativen.

- Klare Kommunikation an die Kommunen bezüglich gesetzeskonformer Möglichkeiten der Depotcontainersammlung sowie pragmatisches Vorgehen vonseiten der Behörden.

- Verstärkter Einsatz von Anreizsystemen, z. B. EKG als Spende.

- Transparentes Monitoring von EAGSammelsystemen als Voraussetzung zur Optimierung.

Um Verluste in der Verwertung zu reduzieren wird vorgeschlagen:

- Verbesserung der Rückgewinnung, vor allem von Kunststoffen im Recycling.

- Engere Kooperation und besserer Informationsaustausch entlang der
Wertschöpfungskette, z. B. zwischen Produzenten, Aufbereitern und Verwertern

- Optimierung der Aufbereitungsprozesse durch Berücksichtigung der Charakteristik der Sammelware.

- Schaffung von Anreizen zur Rückgewinnung speziell von kritischen Rohstoffen, um künftigen Versorgungsengpässen entgegenzuwirken.

Wie anfangs erwähnt, ist das Rückgewinnen von Wertstoffen ein wichtiger Teil des Europäischen Kreislaufwirtschaftspakets. Derzeit werden jedoch trotz hochwertigen Recyclings viele Wertstoffe nicht rückgewonnen, da es sich als nicht wirtschaftlich erweist. Obwohl Verwertung einen wichtigen Beitrag zur Ressourcenschonung liefern kann, kann eine Erhöhung von Sammel- und Verwertungsquoten nicht die einzige Maßnahme zur Umsetzung der europäischen Ambitionen sein. Die Reduktion des Konsums hat - obwohl keine neue Forderung - noch immer nicht jenen Stellenwert bei Produzenten und in der Gesellschaft, der zum langfristigen nachhaltigen Umgang mit Ressourcen nötig wäre. Diesbezügliche Maßnahmen umfassen u. a. die Verlängerung der Nutzungsdauer von Geräten, besseres Produktdesign und neue Geschäftsmodelle. Weiters braucht es eine breite Diskussion, wie die Verwertungskette insgesamt optimiert werden kann, wobei auch die Produktion miteingeschlossen werden muss. Produkte werden immer komplexer und weisen höhere Materialvielfalt auf. Beides sind
Trends, die das Recycling erschweren. Es müssen daher neue Ansätze entwickelt werden, um die einzelnen Akteure aus Produktion, Nutzung und Verwertung besser auf das gemeinsame Ziel der nachhaltigen Ressourcennutzung abzustimmen.

Danksagung Dieses Projekt (Rohstoffe aus EAG) wurde von Dr. Stefan Luidold (Montanuniversität Leoben) geleitet und von der österreichischen Forschungsförderungsgesellschaft (FFG Projektnummer 843620) unterstützt. Besonderer Dank geht an die MüllerGuttenbrunn Gruppe und DI Günther Höggerl für die wertvolle Zusammenarbeit und an DI Iris Gruber für ihre Unterstützung beim Verfassen dieses Artikels.

Funding Open access funding provided by University of Natural Resources and Life Sciences Vienna (BOKU).

Open Access Dieser Artikel wird unter der Creative Commons Namensnennung 4.0 International Lizenz (http:// creativecommons.org/licenses/by/4. $0 /$ deed.de) veröffentlicht, welche die Nutzung, Vervielfältigung, Bearbeitung, Verbreitung und Wiedergabe in jeglichem Medium und Format erlaubt, sofern Sie den/die ursprünglichen $\mathrm{Au}$ tor(en) und die Quelle ordnungsgemäß nennen, einen Link zur Creative Commons Lizenz beifügen und angeben, ob Änderungen vorgenommen wurden.

\section{Literatur}

\begin{abstract}
Abfallwirtschaftsbetrieb Wetterau (2017): Wetteraukreis Spitzenreiter im Recyceln von Elektroaltgeräten - Abfallwirtschaftsbetrieb Wetterau. http://www.awb-wetterau.de/nachrichten/ wetteraukreis-spitzenreiter-im-recyceln-vonelektroaltgeraeten.html. Gesehen 4. Jänner 2017 Beigl, P., Salhofer, S. (2016): Untersuchung der Zusammensetzung von Elektrokleingeräten und Entwicklung einer Demontagedatenbank. In: Pomberger, R., Adam, J., Aldrian, A., Hiden, S., Höllen, D., Kranzinger, L., Lorber, K.E., Neuhold, S., Nigl, T., Pfandl, K., Sarc, R., Schwarz, T., Sedlazeck, P., Wellacher, M. (Hrsg.): DepoTech 2016 Tagungsband zur 13. Recy \& DepoTech Konferenz, Montanuniversität Leoben/Österreich, 8.-11. November 2016

Beigl, P., Schneider, F., Salhofer, S. (2012): Takeback systems for mobile phones: review and recommendations. Proceedings of the Institution of Civil Engineers - Waste and Resource Management 165 (1):25-35. doi:https://doi.org/10.1680/ warm.2012.165.1.25

Bundeskanzleramt (BKA) (2004): Verordnung des Bundesministers für Land- und Forstwirt-
\end{abstract}

schaft, Umwelt und Wasserwirtschaft über Behandlungspflichten von Abfällen (Abfallbehandlungspflichtenverordnung)StF: BGBl. II Nr. 459/2004

Bundeskanzleramt (BKA) (2005): Verordnung des Bundesministers für Land- und Forstwirtschaft, Umwelt und Wasserwirtschaft über die Abfallvermeidung, Sammlung und Behandlung von elektrischen und elektronischen Altgeräten (Elektroaltgeräteverordnung - EAG-VO)

Bundeskanzleramt (BKA) (2014): Verordnung des Bundesministers für Land- und Forstwirtschaft, Umwelt und Wasserwirtschaft, mit der die Elektroaltgeräteverordnung geändert wird (EAG-VO-Novelle 2014)

Bundesamt für Umwelt (BAFU) (2017): Elektrische und elektronische Geräte. https://www. bafu.admin.ch/bafu/de/home/themen/abfall/ abfallwegweiser-a-z/elektrische-und-

elektronische-geraete.html. Gesehen 3. März 2017

Bundesministerium für Umwelt, Naturschutz, Bau und Reaktorsicherheit (BMUB) (2017): ,eSchrott-App' vorgestellt. Wissen, wo der Elek-
tro(nik)-Schrott hingehört. http://www.bmub. bund.de/presse/pressemitteilungen/pm/artikel/ eschrott-app-vorgestellt-wissen-wo-derelektronik-schrott-hingehoert/?tx_ttnews \%2525255BbackPid\%2525255D=2254. Gesehen 3. Jänner 2017

Chancerel, P., Meskers, C.E.M., Hageluken, C., Rotter, V.S. (2009): Assessment of Precious Metal Flows During Preprocessing of Waste Electrical and Electronic Equipment. J Ind Ecol 13 (5):791-810. doi:https://doi.org/10.1111/j.15309290.2009.00171.x

City of Copenhagen (2017): How to handle your household waste? http://international.kk. $\mathrm{dk} /$ artikel/how-handle-your-household-waste. Gesehen 1. März 2017

Deutsche Post (2017): Altes Handy oder Druckerpatronen kostenlos entsorgen. http://www. deutschepost.de/content/dpag/de/e/

electroreturn/verbraucher.html. Gesehen 3. Jänner 2017

EAK (2016): Elektroaltgeräte Koordinierungsstelle Austria - Tätigkeitsbericht 2015. Elektroaltgeräte Koordinierungsstelle Austria GmbH 
Europäische Kommission (2015): Mitteilung der Kommission an das Europäische Parlament, den Rat, den Europäischen Wirtschafts- und Sozialausschuss und den Ausschuss der Regionen: Den Kreislauf schließen - Ein Aktionsplan der EU für die Kreislaufwirtschaft. Europäische Kommission (EK), Brüssel

Europäisches Parlament (2012): Richtlinie 2012/19/EU des Europäischen Parlaments und des Rates vom 4. Juli 2012 über Elektro- und Elektronik-Altgeräte

Eurostat (2017): Waste Electrical and Electronic Equipment (WEEE). In: Eurostat - Your Key Eur. Stat. http://ec.europa.eu/eurostat/web/waste/ key-waste-streams/weee. Gesehen 10. Jänner 2017

Geyer, R., Blass, V.D. (2010): The economics of cell phone reuse and recycling. Int J Adv Manuf Tech 47 (5-8):515-525. doi:https://doi.org/10. 1007/s00170-009-2228-z

Hobohm, J., Wasserberg, S., Kuchta, K., Kuebart, C. (2016): Evaluierung der Sammlung von Elektrokleingeräten über Depotcontainer der Stadtreinigung Hamburg. Müll Abfall 2:83-89.

Höggerl, G. (2015): Aufbereitung von Elektroaltgeräten nach dem neuesten Stand der Technik. BHM Berg- und Hüttenmännische Monatshefte 160, 275-283

Huisman, J., Magalini, F., Kuehr, R. et al (2007): 2008 Review of Directive 2002/96 on Waste Electrical and Electronic Equipment (WEEE), Final Report. United Nations University, Bonn

Kannegaard, J. (2015): City of Copenhagen Waste Management and Recycling.E-mail Kommunikation. Mai 2015

Kiil, A. (2017): Københavns Kommune - Teknik og Miljøforvaltningen. Persönliche Kommunikation. März 2017

Larsen, T.M. (2017): Gemeinde Stavanger. Persönliche Kommunikation. März 2017

Meissner, R. (2017): IVAR IKS, Norwegen. Persönliche Kommunikation. März-Juli 2017

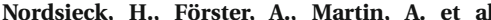
(2016): Sicheres Sammeln von Elektoaltgeräten in Depotcontainersystemen. Universität Augsburg und bifa Umweltinstitut, Augsburg

Oguchi, M., Murakami, S., Sakanakura, H. Kida, A., Kameya, T. (2011): A preliminary categorization of end-of-life electrical and electronic equipment as secondary metal resources. Waste Management 31 (9-10):2150-2160. doi:https:// doi.org/10.1016/j.wasman.2011.05.009

Oguchi, M., Sakanakura, H., Terazono, A. (2013): Toxic metals in WEEE: Characterization and substance flow analysis in waste treatmen processes. Science of the Total Environment 463:1124-1132. doi:https://doi.org/10.1016/j. scitotenv.2012.07.078

RepaNet (2017): Innovatives Sammelsystem für Klein-EAG: Gutscheine für Elektroschrott. In RepaNet. http://www.repanet.at/gutscheinefuer-elektroschrott/. Gesehen 10. Jänner 2017 Salhofer, S., Tesar, M. (2011): Assessment of removal of components containing hazardous substances from small WEEE in Austria. Journa of Hazardous Materials 186 (2-3):1481-1488. doi:https://doi.org/10.1016/j.jhazmat.2010.12. 030

Stadt Salzburg (2017): Elektrogeräte, Elektroaltgeräte. https://www.stadt-salzburg.at/internet/ wirtschaft_umwelt/abfall_abwasser/abfall_a z $\mathrm{z}$ abfall_von_a_z/elektrogeraete_elektroalt

geraete_209735.htm? redirect=404. Gesehen 3 . August 2017

Tomasin, T. (2013): Evaluierung und Vergleich eines Sammelversuchs von Elektroaltgeräten in einer Wiener Wohnhausanlage. Diplomarbeit, Institut für Abfallwirtschaft-Universität für Bodenkultur

Trischler, N., Stadtmüller, U., Deister, U. (2015)

Vorschläge zur Optimierung der Rückgabe von Mobiltelefonen und Smartphones. Müll Abfall 12:684-689
Umweltbundesamt (2017a): Elektro- und Elektronikaltgeräte. In: Umweltbundesamt. http:// www.umweltbundesamt.de/daten/abfallkreislaufwirtschaft/entsorgung-verwertungausgewaehlter-abfallarten/elektro-

elektronikaltgeraete. Gesehen 3. August 2017

Umweltbundesamt (2017b): Elektro- und Elektronikgerätegesetz. http://www.umweltbundes amt.de/themen/abfall-ressourcen/produkt verantwortung-in-der-abfallwirtschaft/ elektroaltgeraete/elektro-elektronikgeraete gesetz. Gesehen 1. März 2017

Unger, N., Beigl, P., Höggerl, G., Salhofer, S. (2017): The greenhouse gas benefit of recycling waste electrical and electronic equipment above the legal minimum requirement: An Austrian LCA case study. Journal of Cleaner Production 164:1635-1644. doi:https://doi.org/10.1016/j. jclepro.2017.06.225

United Nations (2015): ADR European Agreement concerning the International Carriage of Dangerous Goods by Road

Verband kommunaler Unternehmen (VKU) (2017): Die kommunale Erfassung von Elektro- und Elektronikaltgeräten - Praktische Fragen und Antworten. Inforamtion 89. https:// www.vku-shop.de/?utm_source=vku\&utm medium=website\&utm_campaign=info89

Winkel, H. (s.a.): Elektrokleingeräte - Sammlung - Ergebnisse eines Pilotversuches in der Stadt Mainz. Abfallwirtschaftsbetrieb Wetterau. http://www.awb-wetterau.de/files/awb/VKU/ Vortraege\%202013/07_Elektrokleingeraete-

Winkel.pdf. Gesehen 10. Jänner 2017

Winterberg, S. (2013) Erfassungssysteme für Elektroschrott in der Praxis. Müll und Abfall 11 (13):584-586 